\title{
Potenzial von Sentinel-2-Satellitendaten für Anwendungen im Waldbereich
}

Dominique Weber Christian Ginzler Stefan Flückiger Christian Rosset
Hochschule für Agrar-, Forst- und Lebensmittelwissenschaften $(\mathrm{CH})^{*}$

Eidgenössische Forschungsanstalt für Wald, Schnee und Landschaft $(\mathrm{CH})$

Forstbetrieb der Burgergemeinde Bern (CH)

Hochschule für Agrar-, Forst- und Lebensmittelwissenschaften (CH)

\section{Potenzial von Sentinel-2-Satellitendaten für Anwendungen im Waldbereich}

\begin{abstract}
Seit Ende 2015 sind Sentinel-2-Satellitendaten frei erhältlich. Die hohe räumliche Auflösung, die grossflächige Verfügbarkeit sowie die häufige Wiederholung der Aufnahmen in für Vegetationsanalysen wichtigen Spektralbändern eröffnen neue Perspektiven für Anwendungen im Waldbereich. In dieser Studie wurde das Potenzial von Sentinel-2 anhand von Anwendungsbeispielen im Wald des Forstbetriebs der Burgergemeinde Bern (Schweizer Mittelland) untersucht. Hierfür wurden die Ermittlung des Laub- bzw. Nadelholzanteils sowie die Erfassung von forstlichen Eingriffen und natürlichen Störungen getestet. Vom Forstbetrieb erhobene Daten (Stichprobeninventur, kartierte Holzschläge, Waldbrandfläche) wurden als Trainingsdaten und für die Validierung der Ergebnisse verwendet. Mit einem Regressionsmodell, dem Sentinel-2-Daten von drei verschiedenen Zeitpunkten zugrunde lagen, konnten 56\% der Variation des Laub- bzw. Nadelholzanteils erklärt werden. Dieses Modell war deutlich besser als die Modelle, die auf einem oder zwei Aufnahmezeitpunkten beruhten. Die Unterscheidung von reinen Laub- und Nadelholzflächen erreichte eine Genauigkeit von 90\%. Die Detektion von Eingriffen wurde anhand der Unterschiede der spektralen Information aus zwei Zeitpunkten ermittelt und zeigte eine hohe Übereinstimmung mit den Angaben des Forstbetriebs. Dies war auch bei der Erkennung und Abgrenzung der Waldbrandfläche der Fall, was das Potenzial von Sentinel-2-Daten für die zeitnahe und grossflächige Erfassung von natürlichen Störungen unterstreicht.
\end{abstract}

Keywords: Sentinel-2, remote sensing, classification, forest monitoring, change detection doi: $10.3188 /$ szf.2018.0026

* Länggasse 85, CH-3052 Zollikofen, E-Mail dominique.weber@bfh.ch

$\mathrm{F}$ ür eine effiziente, innovative und nachhaltige Waldbewirtschaftung sind Informationen über den Zustand und die Entwicklung des Waldes von zentraler Bedeutung (BAFU 2013). Die Fernerkundung bietet Möglichkeiten und Verfahren, um Daten flächendeckend und zeitnah zu erfassen. Bislang wurden im Waldbereich hochaufgelöste Luftbilder und Daten aus flugzeuggetragenem Laserscanning (auch LiDAR, engl. Light Detection and Ranging, genannt) genutzt. Wesentlich verbessert haben sich in den letzten Jahren aber auch die Datenqualität und die Datenverfügbarkeit von Satellitenbildern (Ginzler \& Waser 2017). Bis anhin setzte bei den grossflächig und frei verfügbaren Satellitenbildern der Landsat-Datensatz der NASA den Standard (Masek et al 2006). Die Auflösung von 30 m limitiert jedoch die Anwendungsmöglichkeiten für die Schweizer Waldwirtschaft. Die Sentinel-2-Mission, die aus den Satelliten Sentinel-2A (ab 2015) und
Sentinel-2B (ab 2017) besteht und Teil des Erdbeobachtungsprogramms «Copernicus» der Europäischen Kommission ist, eröffnet neue Perspektiven und Anwendungsmöglichkeiten: Alle fünf Tage werden Bilder der Schweiz aufgenommen, dies bei einer Schwadbreite (Aufnahmestreifen, engl. swath width) von 290 km, einer räumlichen Auflösung ab 10 m und in für Vegetationsanalysen wichtigen Spektralbändern (Drusch et al 2012). Mit der höheren Auflösung ist auch der Detaillierungsgrad im Vergleich zu Landsat deutlich besser, und er ist so hoch, dass sich die Sentinel-2-Daten auch für Praxisanwendungen im Bereich Waldmonitoring und Waldmanagement eignen (Abbildung 1).

Die beiden Satelliten Sentinel-2A/B erfassen Informationen in 13 verschiedenen Spektralbändern, wobei neben dem sichtbaren Lichtspektrum (Blau, Grün und Rot) speziell die Bereiche im nahen bis kurzwelligen Infrarot eine detaillierte Analyse 


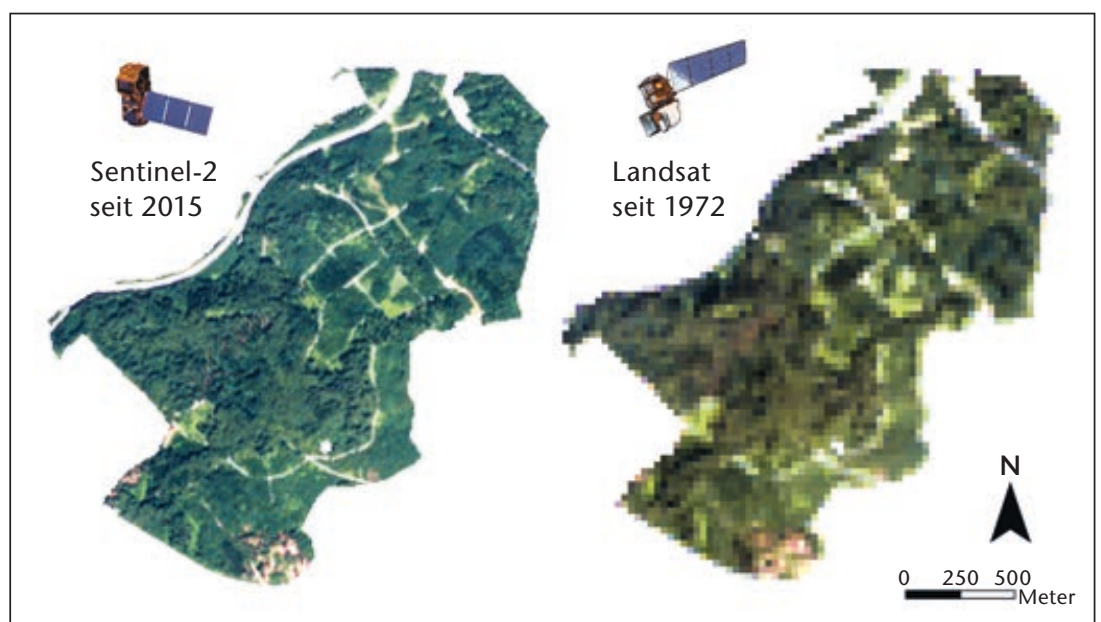

Abb 1 Aufnahme des Könizbergwaldes (BE) mit Sentinel-2A (Auflösung: 10 m; links) und mit Landsat-8 (Auflösung: 30 m; rechts). Beide Aufnahmen stammen vom August 2016 und sind als Echtfarbenbilder ( $R G B)$ wiedergegeben.

des Vegetationszustandes ermöglichen. Die Reflexion der elektromagnetischen Strahlung im roten Spektrum hängt vom Chlorophyllgehalt und der Fotosyntheseaktivität ab. Die Reflexion im nahen Infrarot korreliert mit der Blattstruktur. Das Verhältnis der Reflexionen im roten und nahen infraroten Spektralbereich ist ein Indikator für die Vitalität und die Produktivität von Pflanzen. Mit der Berechnung von Vegetationsindizes wie dem Normalized Difference Vegetation Index (NDVI) können solche Informationen einfach hergeleitet und visualisiert werden (Rouse et al 1974). Zusätzlich bietet Sentinel-2 drei Bänder im sogenannten Red-Edge-Bereich, dem Wellenbereich zwischen 700 und $800 \mathrm{~nm}$ im nahen Infrarot, in welchem sich die Reflexion in Abhängigkeit vom Chlorophyllgehalt der Pflanzen und der Zellstruktur der Blätter schnell ändert. Mit diesen Bändern lassen sich Vitalitätsveränderungen noch genauer erfassen (Delgido et al 2011, Frampton et al 2013).

Ein grosser Mehrwert gegenüber existierenden Fernerkundungsdaten liegt bei Sentinel-2 in der hohen Wiederholungsrate der Aufnahmen. Damit stehen zeitnahe Informationen zur Verfügung, und die Entwicklung bzw. Veränderung der Vegetation innerhalb eines Jahres kann verfolgt werden (Abbildung 2). Die Verfügbarkeit von Bildern zu verschiedenen Jahreszeiten vereinfacht die Unterscheidung von Laub- und Nadelholz sowie von Hauptbaumarten (Immitzer et al 2016, Key 2001, Wolter et al 1995). Phänologische Ereignisse (z.B. Blattaustrieb, Herbstverfärbung, Blütephase) wie auch der Unterschied zwischen Winter- und Sommerzustand können dafür genutzt werden. Zudem bietet die hohe zeitliche Auflösung die Möglichkeit, abrupte Veränderungen (z.B. Holzschläge, Sturmereignisse, Frostschäden, Waldbrände) zeitnah zu erfassen und die langfristige Entwicklung der Vegetation zu verfolgen.

Obwohl Sentinel-2-Daten erst seit Kurzem zur Verfügung stehen, wurde ihr hohes Potenzial bereits von mehreren Studien aufgezeigt, zum Beispiel für die Baumartenklassifikation (Immitzer et al 2016), die Erkennung von Trockenstress (Dotzler et al 2015) und die Erfassung von Waldbrandflächen (Verhegghen et al 2016, Fernández-Manso et al 2016). Die oben erwähnten Studien sind nur bedingt auf die Schweizer Verhältnisse übertragbar (Klima, Bewirtschaftung, Praxistauglichkeit) und wurden teilweise mit Testdatensätzen (Status «pre-operational») oder simulierten Daten durchgeführt. Das Ziel dieser Studie war es, die Möglichkeiten von Sentinel-2 für Anwendungen in der forstlichen Praxis zu testen. Ausgewählt wurden hierfür die Ermittlung des

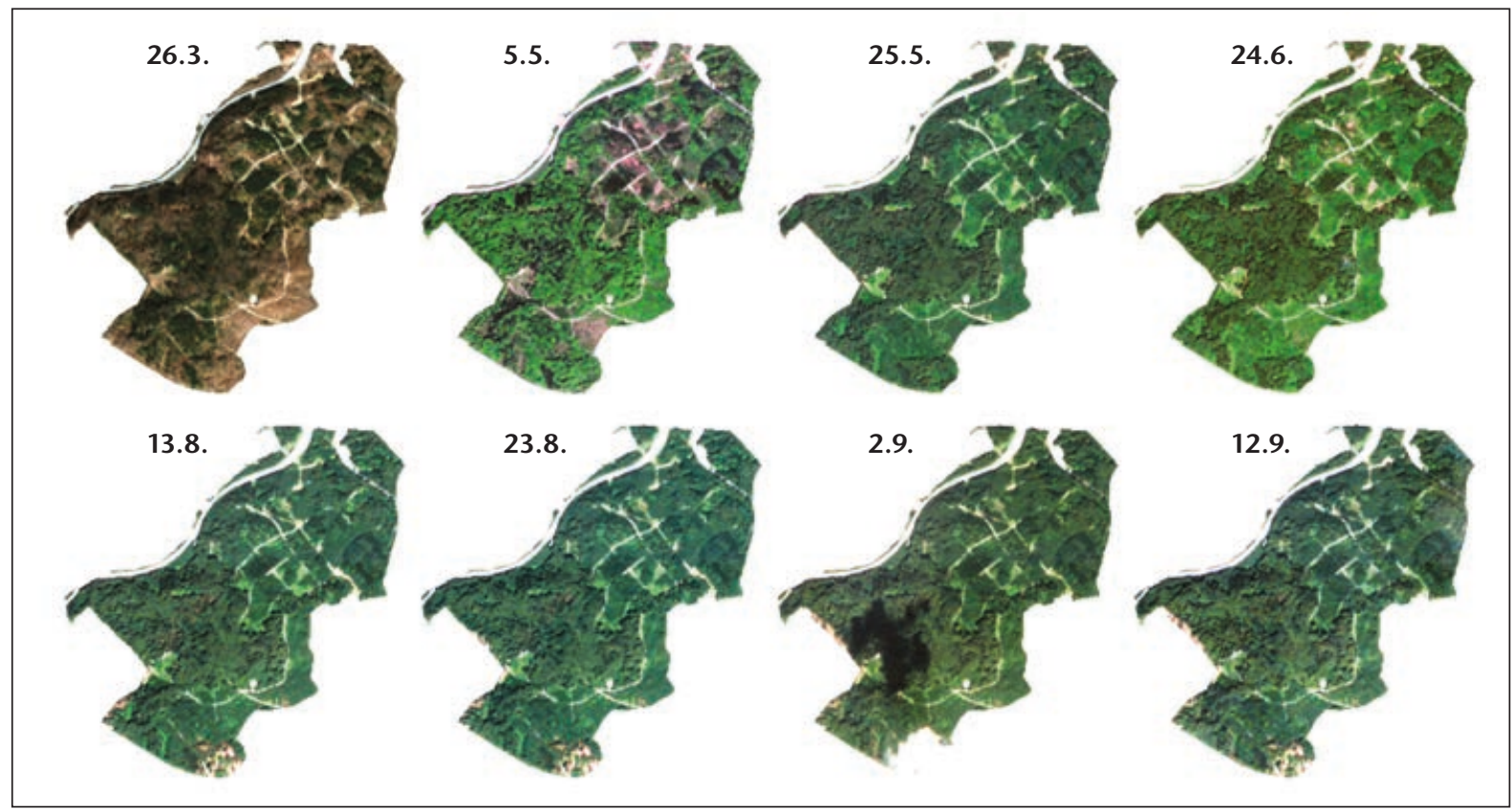

Abb 2 Entwicklung bzw. Veränderung der Vegetation im Jahresverlauf im Könizbergwald (BE) im Jahr 2016. Im Bild vom 2.9. ist eine Wolke bzw. deren Schatten erkennbar. 


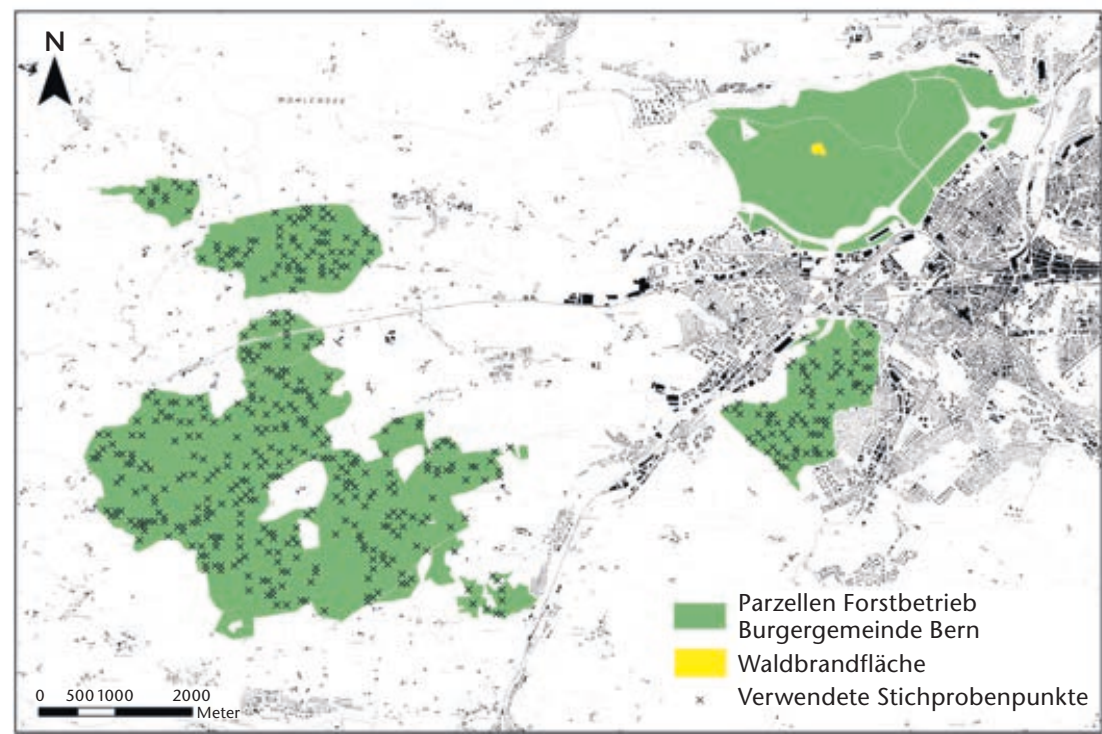

Abb 3 Das Untersuchungsgebiet mit Wäldern des Forstbetriebs der Burgergemeinde Bern. Darin eingetragen sind auch die aus der Betriebsinventur 2016/2017 zufällig ausgewählten Stichprobenpunkte, die als Referenzdatensatz dienten, sowie die analysierte Waldbrandfläche. Übersichtsplan: Amt für Geoinformation des Kantons Bern

Laub- bzw. Nadelholzanteils sowie die zeitnahe Erkennung von Waldveränderungen, die durch die Bewirtschaftung (Holzschläge) und natürliche Störungen (z.B. Waldbrand) hervorgerufen worden sind.

\section{Material und Methoden}

\section{Untersuchungsgebiet und Referenzdaten}

Das Untersuchungsgebiet liegt im Schweizer Mittelland und umfasst verschiedene Wälder des Forstbetriebs der Burgergemeinde Bern (FBB; Abbildung 3). Die analysierten Waldgebiete haben eine Fläche von ca. 2200 Hektaren und werden nach den Prinzipien des schweizerischen Femelschlagbetriebs bewirtschaftet. Für die Kalibrierung und Validierung der statistischen Modelle wurden Ergebnisse der Be- triebsinventur 2016/2017 des FBB verwendet. Diese Stichprobeninventur erfolgte auf einem Raster von $62.5 \mathrm{~m} \times 62.5 \mathrm{~m}$ mit der Smartphone-Applikation MOTI (Rosset et al 2015). Bei der Messung der Grundfläche der Bestandesoberschicht mittels Winkelzählprobe werden die Baumarten differenziert, daraus ergibt sich die Schätzung des Laub- bzw. Nadelholzanteils. Zusätzlich wurden hochaufgelöste Orthofotos (swissimage (c) 2017 swisstopo) sowie Informationen des FBB zu den Schlagflächen und zur vorhandenen Waldbrandfläche für die Interpretation der Resultate hinzugezogen.

\section{Sentinel-2-Daten und Vorbereitung}

Vom Copernicus Open Access Hub ${ }^{1}$ wurden Sentinel-2-Daten von 2016 und 2017 für den entsprechenden Ausschnitt (Kachelnummer 32TLT) als Level 1C, als radiometrisch und geometrisch korrigierte Produkte, heruntergeladen (Drusch et al 2012). Anhand der Sentinel-2-Metadaten wurde der Bewölkungsgrad aller Aufnahmen ermittelt. Dies ermöglichte eine erste Übersicht über die effektiv verfügbaren Daten und die Datenselektion unter Berücksichtigung der jährlichen Vegetationsentwicklung. Für die Analysen wurden alle Bänder mit einer Pixelgrösse von $10 \mathrm{~m}$ und $20 \mathrm{~m}$ verwendet (Abbildung 4). Dazu mussten die 20-m-Bänder auf eine Pixelgrösse von $10 \mathrm{~m}$ umgerechnet werden. Die 60-mBänder wurden nicht berücksichtigt, da diese primär für die Atmosphärenkorrektur und für die Wolkenerkennung dienen.

\section{Datenanalyse und statistische Auswertung Laub- bzw. Nadelholzanteil}

Für die Ermittlung des Laub- bzw. Nadelholzanteils wurden wolkenfreie Sentinel-2-Daten vom 26. März, 5. Mai und 24. Juni 2016 verwendet, um

$1 \mathrm{https} / /$ scihub.copernicus.eu/dhus (20.11.2017)

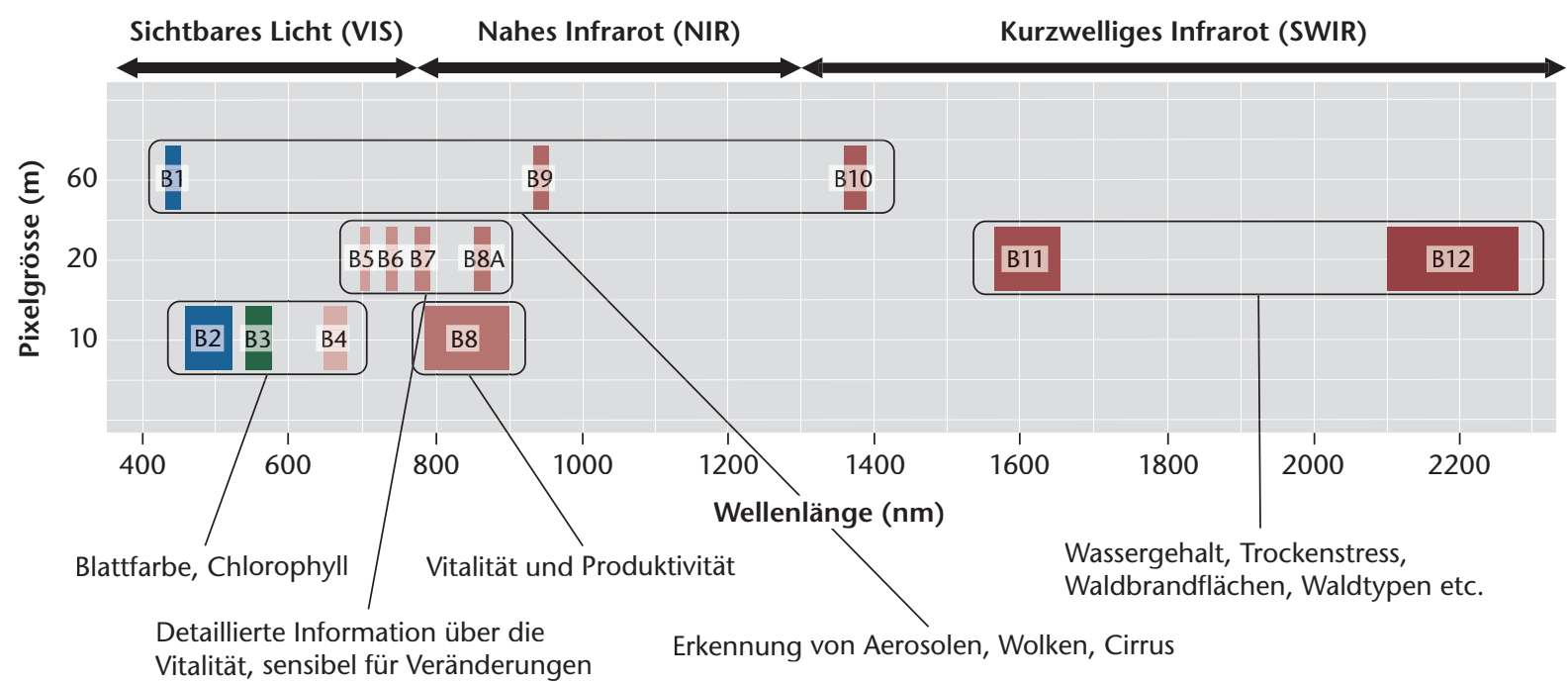

Abb 4 Die 13 Bänder (B) von Sentinel-2 in den verschiedenen Spektralbereichen (Wellenlängen) und ihr Nutzen für die Vegetationsanalyse. 


\begin{tabular}{|l|l|l|}
\hline $\begin{array}{l}\text { Vegetations- } \\
\text { index }\end{array}$ & Formel mit Sentinel-2-Bändern (B) & Referenz \\
\hline $\mathrm{NDVI}$ & $(\mathrm{B} 8-\mathrm{B} 4) /(\mathrm{B} 8+\mathrm{B} 4)$ & Rouse et al $(1974)$ \\
\hline $\mathrm{NDI45}$ & $(\mathrm{B} 5-\mathrm{B} 4) /(\mathrm{B} 5+\mathrm{B} 4)$ & Delegido et al $(2011)$ \\
\hline $\mathrm{MTCl}$ & $(\mathrm{B} 6-\mathrm{B} 5) /(\mathrm{B} 5-\mathrm{B} 4)$ & Dash \& Curran (2004) \\
\hline $\mathrm{MCARI}$ & $\mathrm{B} 5-\mathrm{B} 4-0.2 \times(\mathrm{B} 5-\mathrm{B} 3) \times(\mathrm{B} 5-\mathrm{B} 4)$ & Daughtry $(2000)$ \\
\hline $\mathrm{GNDVI}$ & $(\mathrm{B} 7-\mathrm{B} 3) /(\mathrm{B} 7+\mathrm{B} 3)$ & Gitelson et al $(1996)$ \\
\hline PSSRa & $\mathrm{B} 7 / \mathrm{B} 4$ & Blackburn $(1998)$ \\
\hline S2REP & $705+35 \times(0.5 \times[\mathrm{B} 7+\mathrm{B} 4]-\mathrm{B} 5) /(\mathrm{B} 6-\mathrm{B} 5)$ & Frampton et al $(2013)$ \\
\hline IRECI & $(\mathrm{B} 7-\mathrm{B} 4) /(\mathrm{B} 5 / \mathrm{B} 6)$ & Frampton et al $(2013)$ \\
\hline NBR* & $(\mathrm{B} 8-\mathrm{B} 12) /(\mathrm{B} 8+\mathrm{B} 12)$ & Key \& Benson (2006) \\
\hline
\end{tabular}

Tab 1 Verwendete Vegetationsindizes. Die Auswahl basiert auf Frampton et al (2013) und deckt eine Reihe von etablierten sowie Sentinel-2-spezifischen Indizes ab, welche einen starken Bezug zur Blattfläche bzw. zum Chlorophyllgehalt aufweisen. *Dieser Index wurde nur für die Erkennung der Waldbrandfläche verwendet.

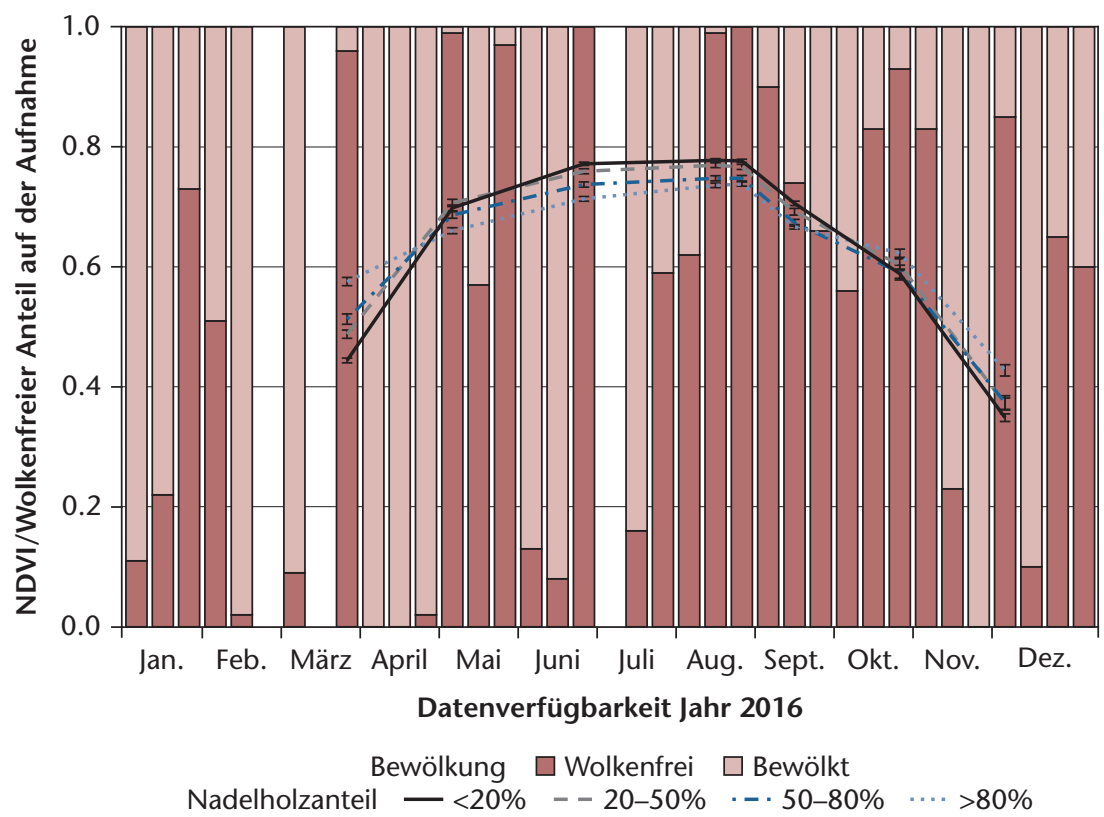

Abb 5 Verfügbarkeit der Sentinel-2-Daten und Bewölkung zum Aufnahmezeitpunkt. Dargestellt ist zudem der Verlauf des Vegetationsindexes NDVI in Beständen unterschiedlichen Mischungsgrades aus der Betriebsinventur des Forstbetriebs der Burgergemeinde Bern (angegebenes Fehlermass: Standardfehler).

wichtige Phasen der Vegetationsentwicklung zwischen Winter und Sommer abzudecken. Neben den originalen Bändern wurden basierend auf der Auswahl von Frampton et al (2013) auch Vegetationsindizes berechnet und als Prädikatoren eingesetzt (Tabelle 1). Die Klassifizierung erfolgte mit Random Forest, einem auf Entscheidungsbäumen basierenden Verfahren (Breiman 2001). Random Forest wurde für die stufenlose Schätzung des Laub- bzw. Nadelholzanteils (Regression) wie auch für die binäre Klassifikation von rein Laubholz ( $>80 \%$ Laubholz) bzw. rein Nadelholz ( $>80 \%$ Nadelholz) verwendet. Als Trainingsdaten für die Modelle wurden Stichprobenpunkte der FBB-Betriebsinventur zufällig ausgewählt - 500 für die Regressionsmodelle und 327 für die binäre Klassifikation. Für die Klassifizierung kam das in R 3.2.3 (R Core Team 2015) implementierte randomForest-Paket (Liaw \& Wiener 2002) mit den Standardeinstellungen (z.B. ntree $=500)$ zur Anwendung. Die Modellgenauigkeit wurde mittels des Out-of-bag-Fehlers ermittelt, der aufgrund des integrierten Bootstrapping einer Validierung mit unabhängigen Referenzdaten entspricht. Die mit einer Pixelgrösse von $10 \mathrm{~m}$ räumlich modellierten Laub- bzw. Nadelholzanteile wurden einem hochaufgelösten Orthofoto gegenübergestellt und visuell überprüft. Zusätzlich wurde mit Random Forest die Wichtigkeit der einzelnen Variablen (Bänder, Vegetationsindizes und Aufnahmezeitpunkte) durch Permutation ermittelt. Durch das Weglassen einer Variablen in allen Entscheidungsbäumen kann die mittlere Abnahme der Modellgenauigkeit bestimmt werden. Als Mass hierfür wurde in den Regressionsmodellen die Zunahme des mittleren quadratischen Fehlers und im Klassifikationsmodell die Zunahme der Fehlerquote verwendet (Breimann 2001).

\section{Waldveränderungen}

Für die Erkennung von Veränderungen (Waldbewirtschaftung, natürliche Störungen) wurden die Unterschiede zwischen zwei zu verschiedenen Zeitpunkten aufgenommenen Sentinel-2-Datensätzen mit geeigneten Vegetationsindizes untersucht. Für forstliche Eingriffe wurde der NDVI verwendet, ein etablierter Index für die Erfassung der Vegetationsaktivität (Tabelle 1; Pettorelli et al 2005). Zwischen den NDVI-Werten vom 19.6.2017 und dem 24.6.2016 wurde die Differenz gebildet $\left(\Delta N D V I=\mathrm{NDVI}_{2017}-\mathrm{NDVI}_{2016}\right)$. Für die zeitnahe Erkennung von natürlichen Störungen wurde der Brand im Bremgartenwald vom 10.4.2017 als Fallbeispiel ausgewählt. Die Normalized Burn Ratio (NBR; Tabelle 1) ist ein für die Erkennung von Waldbrandschäden optimierter Index, da er sensitiv auf Veränderungen der Vegetation und deren Wassergehalt reagiert (Key \& Benson 2006). Detektiert wurde die Waldbrandfläche mittels Differenzbildung zwischen den NBR-Werten vom 10.4.2017 (am Morgen kurz vor dem Brand) und vom 30.4.2017 $\left(\Delta N B R=N R_{\text {vor_Brand }}-\mathrm{NBR}_{\text {nach_Brand }}\right)$. Zur Verifizierung der Resultate wurde die Differenz im jeweiligen Vegetationsindex (forstliche Eingriffe: $\triangle$ NDVI, Waldbrand: $\triangle \mathrm{NBR}$ ) räumlich aufgetragen und mit den Flächenabmessungen, wie sie vom FBB erfasst worden sind, qualitativ verglichen.

\section{Resultate}

\section{Datenverfügbarkeit und Vegetations- entwicklung}

Von theoretisch 37 möglichen Sentinel-2-Aufnahmen der Kachelnummer 32TLT für das Jahr 2016 (alle 10 Tage) waren 34 effektiv verfügbar, 21 hatten eine Bewölkung von weniger als 50\% und 11 eine 


\begin{tabular}{|c|c|c|c|c|c|c|c|c|c|c|c|c|}
\hline & \multicolumn{9}{|c|}{ Regressionsmodelle für Laub- bzw. Nadelholzanteil } & \multicolumn{3}{|c|}{$\begin{array}{c}\text { Klassifikation von } \\
\text { Laub- bzw. Nadelholz }\end{array}$} \\
\hline \multirow[t]{2}{*}{ Modellgüte } & \multicolumn{9}{|c|}{ Erklärte Varianz in \% (pseudo $\mathrm{R}^{2}$ ) } & \multicolumn{3}{|c|}{ Genauigkeit in \% } \\
\hline & \multicolumn{3}{|c|}{$46 \%$} & \multicolumn{3}{|c|}{$51 \%$} & \multicolumn{3}{|c|}{$56 \%$} & \multicolumn{3}{|c|}{$90 \%$} \\
\hline \multirow[t]{11}{*}{ Wichtigste 10 Variablen } & \multicolumn{9}{|c|}{ Zunahme \% MQF durch Permutation } & \multicolumn{3}{|c|}{$\begin{array}{l}\text { Zunahme der Fehlerquote } \\
\text { in } \% \text { durch Permutation }\end{array}$} \\
\hline & März & NDVI & 22.9 & Mai & B3 & 18.0 & März & NDVI & 19.3 & Juni & B11 & 12.3 \\
\hline & März & PSSRa & 20.7 & März & NDVI & 16.8 & März & PSSRa & 18.7 & März & PSSRa & 11.5 \\
\hline & März & B11 & 17.8 & März & PSSRa & 16.7 & Juni & B11 & 16.9 & März & NDVI & 11.3 \\
\hline & März & B4 & 17.5 & Mai & B5 & 14.3 & Mai & B3 & 15.7 & März & IRECI & 8.7 \\
\hline & März & B12 & 15.1 & März & B4 & 14.2 & Juni & NDVI & 11.6 & Mai & $\mathrm{MTCl}$ & 8.7 \\
\hline & März & IRECI & 12.7 & Mai & $\mathrm{MTCl}$ & 12.2 & Juni & GNDVI & 11.6 & Mai & B3 & 8.6 \\
\hline & März & B2 & 11.7 & März & B11 & 11.4 & Mai & B5 & 11.0 & März & GNDVI & 8.4 \\
\hline & März & B5 & 10.7 & März & IRECI & 9.8 & März & B4 & 10.7 & Mai & B5 & 8.1 \\
\hline & März & GNDVI & 9.8 & März & B12 & 9.2 & März & IRECI & 9.0 & Juni & GNDVI & 7.8 \\
\hline & März & $\mathrm{B} 8 \mathrm{~A}$ & 8.8 & Mai & MCARI & 9.3 & Mai & $\mathrm{MTCl}$ & 8.8 & März & B11 & 7.7 \\
\hline
\end{tabular}

Tab 2 Güte und wichtigste Variablen des Regressionsmodells zur stufenlosen Ermittlung des Laub- bzw. Nadelholzanteils und des Klassifikationsmodells für die Bestimmung von reinen Laub- bzw. Nadelholzflächen. Die Modellgüte ist als erklärte Varianz in \% (Regression) bzw. als Genauigkeit in \% gemäss Outof-bag-Fehlerquote (Klassifikation) angegeben. Die Wichtigkeit der einzelnen Variablen ist als die mittlere Zunahme des Modellfehlers (mittlerer quadratischer Fehler [MQF] bzw. Fehlerquote) durch Permutation angegeben, wobei höhere Werte eine grössere Wichtigkeit bedeuten (Liaw \& Wiener 2002).

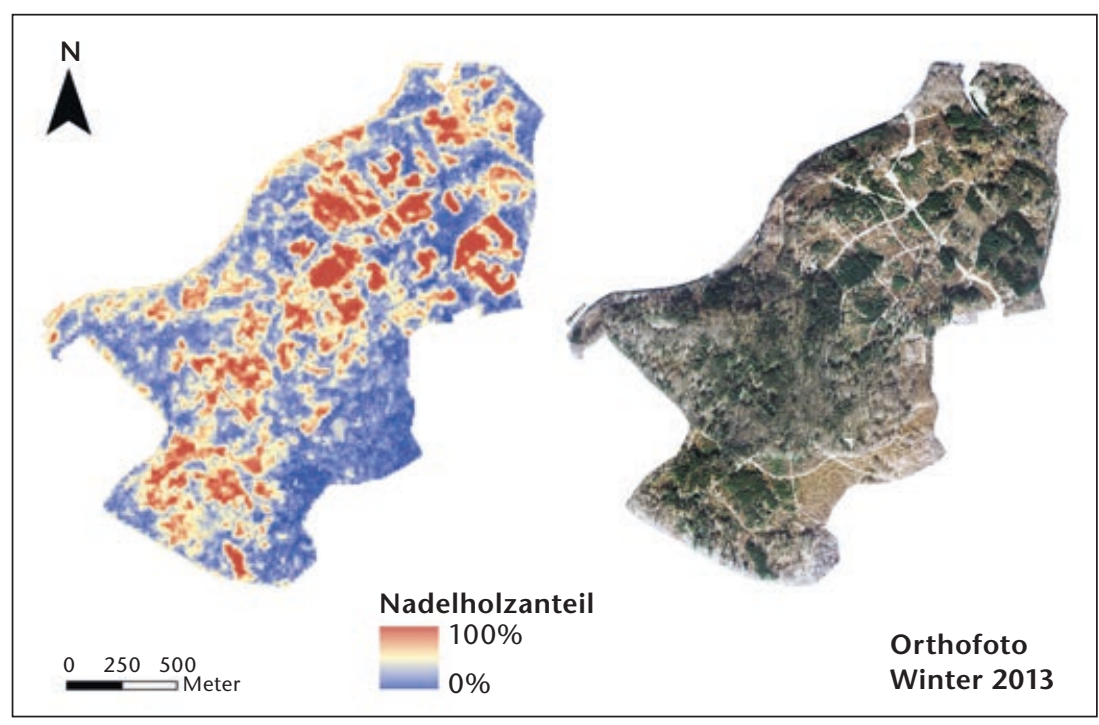

Abb 6 Räumliche Modellierung des Laub- bzw. Nadelholzanteils mit zu drei Zeitpunkten aufgenommenen Sentinel-2-Daten für den Ausschnitt Könizbergwald (links) und hochaufgelöstes Orthofoto des gleichen Ausschnitts zum Vergleich (rechts; swissimage @ 2017 swisstopo).

\section{Laub- bzw. Nadelholzanteil}

Das Regressionsmodell zur stufenlosen Bestimmung des Laub- bzw. Nadelholzanteils erklärte 56\% der Variation der Referenzdaten (pseudo $\mathrm{R}^{2}$ ), wenn zu drei verschiedenen Zeitpunkten aufgenommene Sentinel-2-Daten verwendet wurden (Tabelle 2). Das Modell ist wesentlich besser, als wenn Sentinel-2-Daten von einem (nur März; 46\%) oder zwei Zeitpunkten (März und Mai; 51\%) verwendet worden wären. Das Regressionsmodell zeigt eine gute Übereinstimmung mit der auf dem Orthofoto erkennbaren Verbreitung der Laub- und Nadelhölzer (Abbildung 6). Mit dem Klassifikationsmodell zur Ermittlung von reinen Laub- und Nadelholzflächen wurde eine Genauigkeit von 90\% erzielt. Wichtige spektrale Informationen stammen sowohl beim Regressionsmodell als auch beim Klassifikationsmodell aus allen drei Zeitpunkten, von einzelnen Bändern wie auch von verschiedenen Vegetationsindizes, wobei der NDVI und der PSSRa besonders häufig unter den wichtigsten Variablen erschienen.

Bewölkung von weniger als 20\% (Abbildung 5). Komplett wolkenfrei für das Untersuchungsgebiet waren acht Aufnahmen (in Abbildung 5 alle Zeitpunkte mit NDVI-Werten). Die Entwicklung des NDVI im Jahresverlauf zeigte, dass unterschiedliche Mischungsgrade von Laub- und Nadelholz am besten im Winter und Frühsommer erkennbar sind (Abbildung 5). Laub- wie auch Nadelhölzer zeigen gemäss NDVI eine Zunahme der Vegetationsaktivität im Frühling und eine Abnahme im Herbst, wobei dieser Verlauf für Laubhölzer stärker ausgeprägt ist.

\section{Waldveränderungen \\ Waldbewirtschaftung}

Starke Veränderungen im NDVI zwischen Juni 2016 und Juni 2017 sind in der räumlichen Visualisierung gut erkennbar (Abbildung 7). Eine Zunahme des NDVI beschreibt Flächen mit einer höheren Vegetationsaktivität im Juni 2017, also zum Beispiel Flächen, auf denen im Winter 2015/2016 eingegriffen wurde und die in der Folge einen Vegetationszuwachs aufwiesen. Dagegen beschreibt eine Abnahme des NDVI Flächen, auf welchen beispielsweise im 


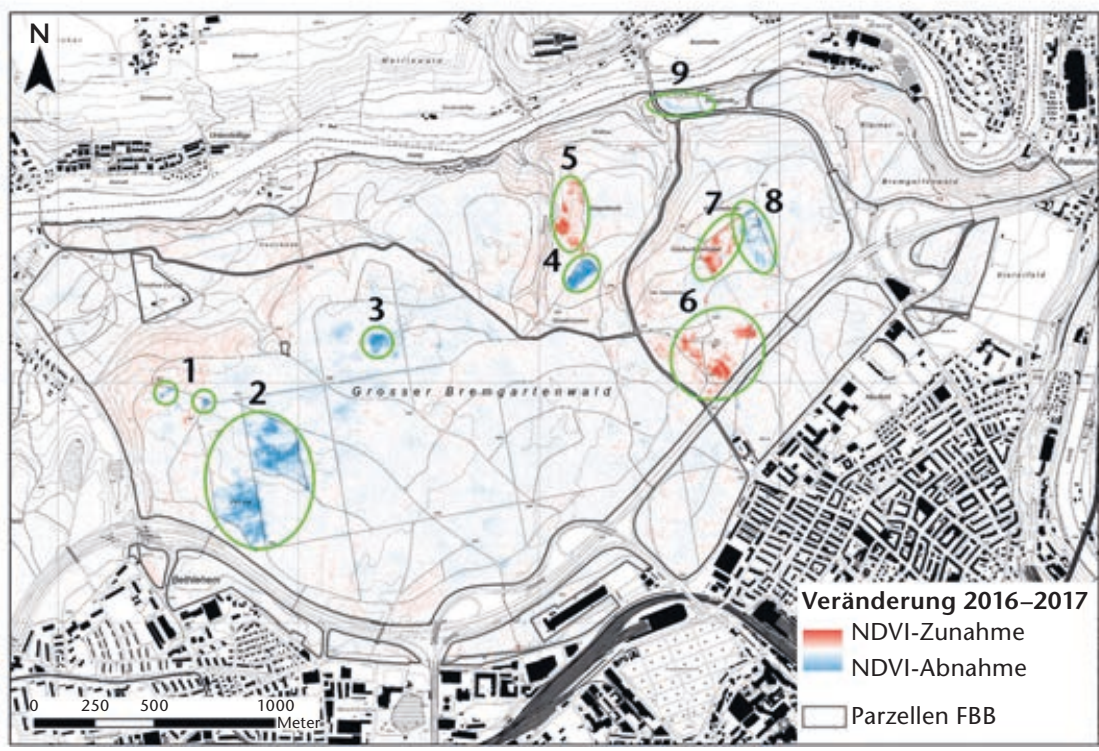

Abb 7 Veränderung des Vegetationsindex NDVI zwischen Juni 2016 und Juni 2017 im Bremgartenwald. Sie entspricht den folgenden, vom Forstbetrieb der Burgergemeinde Bern (FBB) aufgezeichneten Eingriffen und Störungen: Zwangsnutzungen (1,9), Abräumungen (2, 4, 5, 8), Waldbrandfläche (3), Durchforstung (6), Eichenpflanzung seit 2001 (7). Übersichtsplan: Amt für Geoinformation des Kantons Bern

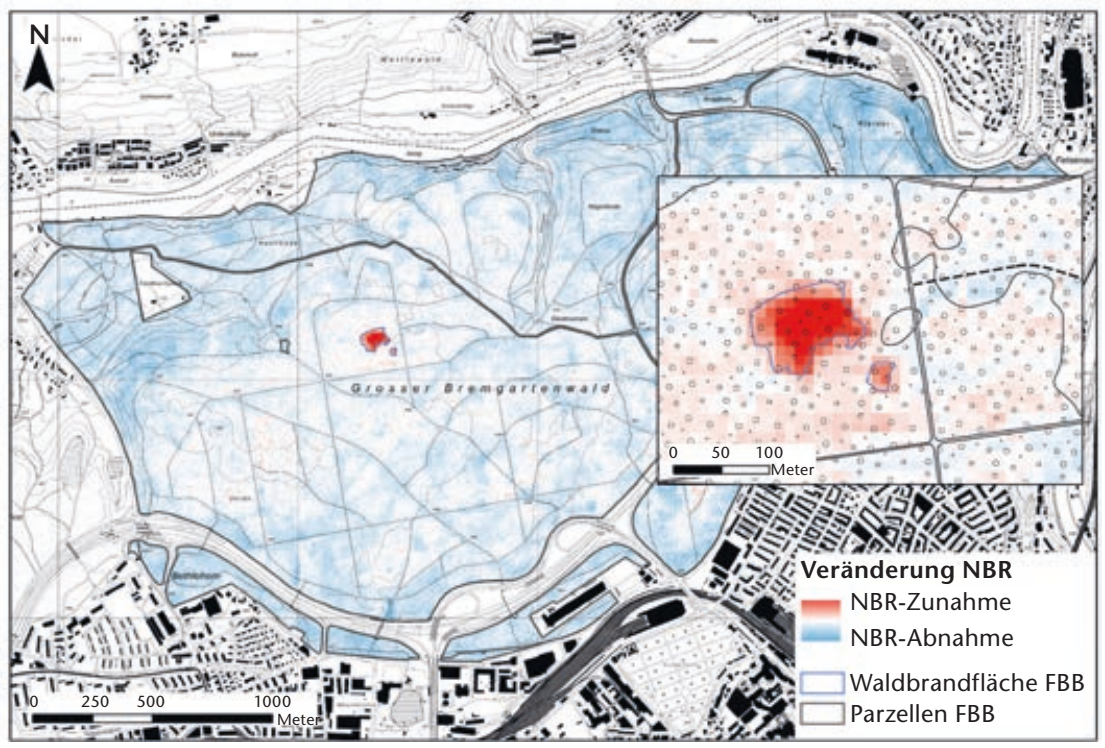

Abb 8 Veränderung der Normalized Burn Ratio (NBR) zwischen dem 10.4. und dem 30.4.2017 im Bremgartenwald. Die vom Waldbrand betroffene Fläche tritt deutlich hervor und stimmt mit der Feldaufnahme des Forstbetriebs der Burgergemeinde Bern (FBB) überein. Übersichtsplan: Amt für Geoinformation des Kantons Bern

Winter 2016/2017 eingegriffen wurde. Die mit Sentinel-2 ermittelten Veränderungen waren für den FBB gut nachvollziehbar und zeigten eine hohe Übereinstimmung mit den von ihm erfassten Eingriffen. Verschiedene Eingriffstypen wie Abräumung, Durchforstung, Anlage von Rückegassen, Waldrandpflege sowie Zwangsnutzungen konnten visuell erkannt werden. Die Unterscheidung der Typen nach rein optischen Kriterien war jedoch nicht möglich.

\section{Natürliche Störungen}

Die Waldbrandfläche im Bremgartenwald ist als die Differenz der NBR vor und nach dem Brand gut erkennbar und zeigte eine hohe Übereinstim- mung mit der Feldaufnahme des FBB (Abbildung 8). Die höchste Zunahme der NBR von +0.75 wurde im Zentrum der Waldbrandfläche ermittelt.

\section{Diskussion}

\section{Datenverfügbarkeit und Vegetations- entwicklung}

Wolken reduzierten die Anzahl der effektiv nutzbaren Sentinel-2-Aufnahmen für das Untersuchungsgebiet im Jahr 2016 stark. Mit einem Aufnahmezyklus von zehn Tagen kann mit jährlich 37 Aufnahmen gerechnet werden, wobei in unserem Fall 20 bis 50\% davon effektiv nutzbar waren. Mit dem zweiten Satelliten, Sentinel-2B, erhöht sich ab Mitte 2017 die Datenverfügbarkeit und damit auch die Wahrscheinlichkeit von wolkenfreien Szenen. Um Einschränkungen in der Datenverfügbarkeit auf Szenenebene weiter zu reduzieren, sollten auch pixelbasierte Ansätze geprüft werden (Hermosilla et al 2016). Damit könnten unabhängig vom Bewölkungsgrad der Szene alle wolkenfreien Pixel oder aus verschiedenen Zeitpunkten generierte Komposite verwendet werden. Die dafür benötigten Masken zur Wolkenerkennung sind Bestandteil der Sentinel2-Produkte oder können mit externen Algorithmen generiert werden (Zhu et al 2015). Dazu kommt, dass für einen Grossteil des Schweizer Mittellandes Daten aus zwei überlappenden Orbits bezogen werden können, was bedeutet, dass gar alle zwei bis drei Tage Daten zur Verfügung stehen.

Eine hohe zeitliche Auflösung ermöglicht es, entscheidende Phasen der Vegetationsentwicklung zu erfassen. Am Beispiel des NDVI-Verlaufes konnte aufgezeigt werden, welche Zeitpunkte sich für die Unterscheidung von Laub- und Nadelholz am besten eignen und wie dies mit der Verfügbarkeit von wolkenfreien Bildern übereinstimmt. Zu beachten ist, dass der Verlauf des NDVI nicht nur vom Mischungsverhältnis von Laub- und Nadelholz, sondern auch von Faktoren wie dem Alter der Bäume oder dem Deckungsgrad abhängig ist (Fassnacht et al 2016). Zudem werden die NDVI-Werte durch forstliche Eingriffe und natürliche Störungen beeinflusst. Bei der in den Satellitendaten gespeicherten Information handelt es sich immer um die Summe der Reflexionen aus dem Kronendach, den tieferen Vegetationsschichten und dem Boden. So ist zum Beispiel der NDVI-Anstieg im Frühling in Laubholzbeständen auch auf die Entwicklung der krautigen Vegetation unter dem Kronendach zurückzuführen, insbesondere bei Beständen mit geringem Schlussgrad.

\section{Laub- bzw. Nadelholzanteil}

Die bei der binären Klassifikation von reinen Laub- bzw. Nadelholzflächen erzielte Genauigkeit 
von 90\% ist vergleichbar mit anderen Studien (Wolter et al 1995, Dalponte et al 2012, Waser et al 2017). Bei der stufenlosen Ermittlung des Laub- bzw. Nadelholzanteils mit dem Regressionsmodell war die Genauigkeit mit 56\% geringer. Das dürfte dem Umstand geschuldet sein, dass beim Klassifikationsmodell nur zwischen reinen Laub- und Nadelholzflächen unterschieden wurde, beim Regressionsmodell hingegen das effektive Mischungsverhältnis die Zielgrösse war. Zudem bezieht sich der Mischungsgrad bei den Referenzdaten auf den Anteil der Laub- und Nadelbäume an der Grundfläche, mit den Fernerkundungsdaten aber wird der Kronendeckungsgrad erfasst. Da Laubbäume bei gleichem Stammdurchmesser eine grössere Kronenfläche haben, wird durch die Fernerkundungsdaten ein höherer Laubholzanteil ausgewiesen, was zu Modellungenauigkeiten führt.

Basierend auf der hier beschriebenen Methode wurde der Laub- bzw. Nadelholzanteil in Projekten in Zusammenarbeit mit dem Amt für Wald des Kantons Bern, dessen Staatsforstbetrieb (SFB) und dem FBB bereits auf grosser Fläche ermittelt. Die Resultate wurden im Feld (u.a. auch in zehn Waldeinheiten des SFB) validiert und zeigten eine für die Praxis brauchbare Genauigkeit. Informationen zum Mischungsverhältnis können somit grossflächig mit relativ geringem Aufwand ermittelt werden und zum Bespiel für die Hiebsatzberechnung wichtige Eingangsgrössen liefern.

Dank der hohen zeitlichen Auflösung von Sentinel-2 sind weitere Verbesserungen bei der Ermittlung des Laub- bzw. Nadelholzanteils vorskizziert: So dürfte für jede beliebige Region der Schweiz ein wolkenfreies Winterbild zu finden sein, das eine Klassifizierung aufgrund des Vegetationszustandes (belaubt/nicht belaubt) ermöglicht. Werden weitere Bilder mit der Vegetationsentwicklung im Frühling und Sommer hinzugenommen, könnte die Unterscheidung noch verfeinert werden. Detaillierte Informationen zur Phänologie könnten auch für die Klassifikation der (Haupt-)Baumarten entscheidend sein (Fassnacht et al 2016, Immitzer et al 2016), nicht zuletzt für jene der Lärche, deren spektrale Signatur derjenigen der Laubhölzer gleicht (Dalponte et al 2012). Eine in der Fernerkundung verbreitete Fehlerquelle sind Schatten aufgrund der Topografie. Mit der Auswahl von Seninel-2-Sommerbildern zum höchsten Sonnenstand könnte diese Fehlerquelle eingedämmt werden. Allerdings bleiben Klassifikationen an Nordhängen in den Bergen, insbesondere in Winterszenen mit tief stehender Sonne, eine Herausforderung.

\section{Waldveränderungen \\ Waldbewirtschaftung}

Die mittels Differenzbildung zwischen den NDVI-Werten aus zwei Zeitpunkten erkannten Ve- getationsveränderungen stimmten gut mit den Nutzungsangaben des FBB überein. Für grössere Forstbetriebe oder kantonale Verwaltungen könnten so grobe Informationen zur Nutzung mit jährlicher Auflösung grossflächig und effizient bereitgestellt werden. Unabhängig davon, ob auf Betriebsebene die Nutzung bereits detailliert erfasst wird, bieten Sentinel-2-Daten die Basis für eine objektive Einschätzung und ein Monitoring der forstlichen Eingriffe. Die Verfügbarkeit von Sentinel-2-Daten mit vergleichbarem Vegetationszustand in aufeinanderfolgenden Jahren ist hierfür entscheidend. Selbst feine und kleinräumige Veränderungen lassen sich so erkennen. Ohne ausreichende Referenzdaten sind allerdings die Interpretation der Veränderungen und damit auch die automatisierte Zuordnung zu Eingriffstypen schwierig. Der Einbezug von dichten Zeitreihen sowie von zusätzlichen Spektralbändern und Vegetationsindizes könnte hier weitere Verbesserungen bringen.

\section{Natürliche Störungen}

Am Beispiel der Brandfläche im Bremgartenwald konnte aufgezeigt werden, dass mit Sentinel2-Daten durch natürliche Störungen verursachte starke Waldveränderungen zeitnah erkannt werden können. Zahlreiche spezifische Indizes und Bandkombinationen stehen bereits zur Verfügung, die für die Erkennung von verschiedenen Typen von Störungen eingesetzt werden können. Der Zeitpunkt der Bildaufnahme ist für die Störungsdetektion entscheidend (Gärtner et al 2016). Die mit Sentinel-2 alle fünf Tage für die ganze Schweiz erfassten Daten stellen diesbezüglich eine ausgezeichnete Grundlage dar. Entsprechend sollte ihr Potenzial für das rasche Schaffen von Übersichten nach landesweiten Sturmschäden geprüft werden. Probleme in diesem $\mathrm{Zu}$ sammenhang können Wolken darstellen, aber auch der Umstand, dass die oft im Winter auftretenden Sturmschäden bei Bäumen im laublosen Zustand schwieriger zu detektieren sind.

\section{Schlussfolgerungen und Ausblick}

Die Resultate dieser Studie zeigen, dass aus Sentinel-2-Daten wichtige Informationen über den Zustand und die Veränderung des Waldes in einer für die Praxis zweckmässigen Genauigkeit und Auflösung abgeleitet werden können. Damit erweitert Sentinel-2 die Palette der Fernerkundungsmethoden im Waldbereich.

Sentinel-2-Daten sind erst seit Kurzem operationell verfügbar. Es ist davon auszugehen, dass mit der fortschreitenden Aufnahme von Bildern sich weitere Anwendungsmöglichkeiten eröffnen. So könnte zum Beispiel der über mehrere Jahre aufgezeichnete Verlauf der Spektralwerte eine Basis sein, um im 
Waldmonitoring die Dynamik des Waldes zu verfolgen, Trends zu erkennen und bedeutende Veränderungen automatisch zu erfassen. Mit jedem Jahr kann auch das Wissen über die Phänologie der Baumarten verbessert werden, was deren automatische Erkennung unterstützt. Dazu kommt, dass der gesicherte, kontinuierliche Datenzufluss und die sich abzeichnenden Automatisierungen es ermöglichen, Bestandesattribute auf effiziente Weise laufend nachzuführen.

Es gilt zu berücksichtigen, dass viele auf Sentinel-2-Daten beruhende Analysen nicht ohne terrestrische Referenzdaten durchgeführt werden können und dass Wolken und deren Schatten die effektiv zur Verfügung stehenden Daten deutlich reduzieren. Der Schattenwurf in steilem Gelände bleibt auch mit Sentinel-2 eine Herausforderung. Es gilt, das Potenzial der Sentinel-2-Daten mit methodischen Untersuchungen und Fallbeispielen weiter zu evaluieren und den Fachspezialisten in der Praxis Werkzeuge in die Hand zu geben, damit sie die Daten im forstlichen Alltag selber nutzen können.

Eingereicht: 10. August 2017, akzeptiert (mit Review): 27. November 2017

\section{Dank}

Wir bedanken uns bei der Burgergemeinde Bern und deren Forstbetrieb für die zur Verfügung gestellten Daten sowie beim Amt für Wald des Kantons Bern und dessen Staatsforstbetrieb für die Validierung der Laub- bzw. Nadelholzanteile im Feld.

\section{Literatur}

BAFU (2013) Waldpolitik 2020. Visionen, Ziele und Massnahmen für eine nachhaltige Bewirtschaftung des Schweizer Waldes. Bern: Bundesamt Umwelt. 66 p.

BLACKBURN GA (1998) Quantifying chlorophylls and carotenoids at leaf and canopy scales. Remote Sens Environ 66: 273-285. BREIMAN L (2001) Random forests. Mach Learn 45: 5-32.

DALPONTE M, BRUZZONE L, GIANELLE D (2012) Tree species classification in the Southern Alps based on the fusion of very high geometrical resolution multispectral/hyperspectral images and LiDAR data. Remote Sens Environ 123: 258-270.

DASH J, CURRAN PJ (2004) The MERIS terrestrial chlorophyll index. Int J Remote Sens 25: 5403-5413.

DAUGHTRY C (2000) Estimating corn leaf chlorophyll concentration from leaf and canopy reflectance. Remote Sens Environ 74: 229-239.

DELEGIDO J, VERRELST J, ALONSO L, MORENO J (2011) Evaluation of Sentinel-2 red-edge bands for empirical estimation of green LAI and chlorophyll content. Sensors 11: 7063-7081.

DOTZLER S, HILL J, BUDDENBAUM H, STOFFELS J (2015) The potential of EnMAP and Sentinel-2 Data for detecting drought stress phenomena in deciduous forest communities. Remote Sens 7: 14227-14258.

DRUSCH M, DEL BELLO U, CARLIER S, COLIN O, FERNANDEZ V, GASCON F ET AL (2012) Sentinel-2: ESA's optical high-resolution mission for GMES operational services. Remote Sens Environ 120: 25-36.
FASSNACHT FE, LATIFI H, STEREŃCZAK K, MODZELEWSKA A, LEFSKY M ET AL (2016) Review of studies on tree species classification from remotely sensed data. Remote Sens Environ 186: 64-87.

FERNÁNDEZ-MANSO A, FERNÁNDEZ-MANSO O, QUINTANO C (2016) SENTINEL-2A red-edge spectral indices suitability for discriminating burn severity. Int J Appl Earth Obs Geoinf 50: 170-175.

FRAMPTON WJ, DASH J, WATMOUGH G, MILTON EJ (2013) Evaluating the capabilities of Sentinel-2 for quantitative estimation of biophysical variables in vegetation. ISPRS J Photogramm Remote Sens 82: 83-92.

GÄRTNER P, FÖRSTER M, KLEINSCHMIT B (2016) The benefit of synthetically generated RapidEye and Landsat 8 data fusion time series for riparian forest disturbance monitoring. Remote Sens Environ 177: 237-247.

GINZLER C, WASER LT (2017) Entwicklungen im Bereich der Fernerkundung für forstliche Anwendungen. Schweiz Z Forstwes 168: 118-126. doi: 10.3188/szf.2017.0118

GITELSON AA, KAUFMAN YJ, MERZLYAK MN (1996) Use of a green channel in remote sensing of global vegetation from EOSMODIS. Remote Sens Environ 58: 289-298.

HERMOSILLA T, WULDER MA, WHITE JC, COOPS NC, HOBART GW ET AL (2016) Mass data processing of time series Landsat imagery. Pixels to data products for forest monitoring. Int J Dig Earth 9: 1035-1054.

IMMITZER M, VUOLO F, ATZBERGER C (2016) First experience with Sentinel-2 data for crop and tree species classifications in Central Europe. Remote Sens 8: 166.

KEY CH, BENSON NC (2006) Landscape assessment: Ground measure of severity, the Composite Burn Index; and remote sensing of severity, the Normalized Burn Ratio. In: Lutes DC, Keane RE, Caratti JF, Key CH, Benson NC et al, editors. FIREMON: Fire Effects Monitoring and Inventory System. Fort Collins CO: USDA Forest Service, Rocky Mountain Research Station, Gen Tech Rep RMRS-GTR-164-CD. pp. LA1-LA51.

KEY T (2001) A comparison of multispectral and multitemporal information in high spatial resolution imagery for classification of individual tree species in a temperate hardwood forest. Remote Sensin Environ 75: 100-112.

LIAW A, WIENER M (2002) Classification and regression by randomForest. R News 2 (3): 18-22.

MASEK JG, VERMOTE EF, SALEOUS NE, WOLFE R, HALL FG ET AL (2006) A Landsat surface reflectance dataset for North America, 1990-2000. IEEE Geosci Remote Sens Lett 3: 68-72.

PETTORELLI N, VIK JO, MYSTERUD A, GAILLARD JM, TUCKER CJ ET AL (2005) Using the satellite-derived NDVI to assess ecological responses to environmental change. Trends Ecol Evol 20: 503-510.

R CORE TEAM (2015) R version 3.2.3. Vienna: R Core Team. WWw.R-project.org/ (21.11.2017).

ROSSET C, BRAND R, WEBER D, WUILLEMIN ERIC, GOLLUT C ET AL (2015) MOTI - ein Tool für die Waldinventur im Taschenformat. Wald Holz 96 (8): 45-48.

ROUSE JW JR, HAAS RH, SCHELL JA, DEERING DW (1974) Monitoring vegetation systems in the Great Plains with Erts. In: Freden SC, Mercanti EP, Becker MA, editors. Third Earth Resources Technology Satellite-1 Symposium. Washington DC: National Aeronautics and Space Administration, NASA SP-351. pp. 309-317.

VERHEGGHEN A, HUGH E, CECCHERINI G, ACHARD F, GOND V ET AL (2016) The potential of Sentinel satellites for burnt area mapping and monitoring in the Congo Basin forests. Remote Sens 8: 986.

WASER LT, GINZLER C, REHUSH N (2017) Wall-to-wall tree type mapping from countrywide airborne remote sensing surveys. Remote Sens 9: 766. 
WOLTER PT, MLADENOFF DJ, HOST GE, CROW TR (1995) Improved forest classification in the Northern Lake States using multi-temporal Landsat imagery. Photogramm Engin Remote Sens 61: 1129-1143.

\section{Le potentiel d'utilisation des données satellites Sentinel-2 dans le domaine forestier}

Les données satellites Sentinel-2 sont disponibles gratuitement depuis fin 2015. Ces données offrent de nouvelles perspectives d'utilisation dans le domaine forestier par leur haute résolution spatiale, leur couverture globale, la fréquence élevée des relevés et le fait qu'elles restituent les canaux spectraux essentiels aux analyses de végétation. Cette étude explore le potentiel des données Sentinel-2 à l'aide d'exemples d'utilisation dans l'entreprise forestière de la Bourgoisie de Berne sur le Plateau suisse. Ont été testés: la détermination de la proportion de feuillus resp. résineux, la localisation d'interventions sylvicoles ainsi que la reconnaissance d'autres perturbations du couvert forestier. Différentes données rassemblées parl'entreprise forestière (inventaire par échantillonnage, carte des coupes de bois, délimitation d'un incendie) ont été utilisées comme données d'entraînement et pour la validation des résultats. $56 \%$ de la variation de la proportion de feuillus resp. résineux ont pu être expliqués à l'aide d'un modèle de régression élaboré sur la base de trois relevés Sentinel-2. Ce modèle a été meilleur que ceux établis sur la base d'une ou deux dates de relévés par le satellite. Les peuplements purs de feuillus et de résineux ont été identifiés avec une précision de $90 \%$. Les interventions sylvicoles ont quant à elles été détectées sur la base des différences entre les informations spectrales de deux relevés Sentinel-2. Les résultats obtenus coïncident en grande partie avec les indications fournies par l'entreprise forestière. II en va de même pour la surface touchée par un incendie, ce qui souligne le potentiel des données Sentinel-2 pour la détection rapide et à large échelle de perturbations du couvert forestier.
ZHU Z, WANG S, WOODCOCK CE (2015) Improvement and expansion of the Fmask algorithm. Cloud, cloud shadow, and snow detection for Landsats 4-7, 8, and Sentinel 2 images. Remote Sens Environ 159: 269-277.

\section{The potential of Sentinel-2 satellite data for forest applications}

Sentinel-2 satellite data have been freely available since the end of 2015. The high spatial resolution, the global coverage, the frequent revisit times and the important spectral bands for vegetation analyses open up new perspectives for forest applications. In this study, the potential of Sentinel-2 was investigated through application examples in the forest enterprise of Burgergemeinde Bern on the Swiss Plateau. For this purpose the determination of the deciduous or coniferous proportion, the recording of interventions, as well as the early detection of natural disturbances were tested. Reference data from the forest enterprise (forest inventory, map of harvesting measures, delimitation of a wildfire) were used as training data and for the validation of the results. By using Sentinel-2 data from three different dates, $56 \%$ of the variation of the deciduous or coniferous proportion could be explained by a regression model. This model was much better than those that used data from one or two time steps only. The distinction of pure deciduous and coniferous stands achieved an accuracy of $90 \%$. The detection of interventions determined by the differences in the spectral information of Sentinel-2 data from two acquisition dates was highly compliant with the information from the forest enterprise. This was also confirmed by the detection of a forest fire area, which highlights the potential of Sentinel-2 for the early and large-scale detection of natural disturbances. 